\title{
As contribuições do trabalho interprofissional no contexto dos serviços de saúde ofertados em uma Unidade de Saúde do município de Benevides no estado do Pará
}

\author{
Alessandra dos Santos Tavares Vieira, Liliane Silva do Nascimento, Nayane Adrielle de Arruda \\ Gomes, Samara de Brito Silva, Michelle Castro da Silva Holanda
}

\begin{abstract}
Resumo
Introdução: O Sistema Único de Saúde (SUS) preconiza uma atenção à saúde considerando o contexto e todos os níveis de atenção para promoção, proteção e recuperação, priorizando o caráter preventivo. Em virtude das transformações no cenário de saúde no Brasil, houve uma reorganização das ações de saúde na atenção básica, inserindo ações de saúde bucal a equipe. Sendo assim os profissionais não devem apenas contribuir para que os usuários adquiram conhecimentos sobre a saúde, mas sim da influência dos cuidados com a mesma sobre a saúde de forma integral. Objetivos: Relatar a experiência da ação educativa interdisciplinar sobre saúde bucal com as crianças e seus familiares usuários da unidade de saúde da família no bairro Santos Dumont, Benevides-PA. Métodos: Trata-se de um relato de experiência vivenciado pela equipe que compõe a unidade de saúde. Esta ação foi pensada em virtude de no acolhimento da demanda assistida uma elevada quantidade de crianças em necessidade de assistência odontológica percebida durante os atendimentos clínicos. Sendo denominada "Venha aprender a cuidar dos primeiros dentinhos". Participaram da atividade além dos profissionais da unidade (auxiliares administrativos, técnico de enfermagem e agentes comunitários de saúde) 26 pessoas, entre crianças e familiares das mesmas. Resultados: Possibilitou o desenvolvimento da prática a partir da interprofissionalidade, com atividades de educação em saúde bucal com as crianças e seus familiares usuários da unidade de saúde, a qual percebeu-se a importância e o impacto que essa conversa educativa proporciona. Haja vista que houve interação de todos durante toda a ação, as crianças e seus responsáveis, além dos próprios profissionais da unidade. Conclusões: É gratificante perceber o trabalho interprofissional determina metodologias motivantes, tornando-se mais proveitoso, pois favorece a criação de vínculo, fortalece a corresponsabilidade e valoriza o autocuidado em saúde de forma integral.
\end{abstract}

Descritores: Educação em saúde, Interdisciplinaridade, Saúde bucal, Atenção básica 